\title{
Expression of the cancer stem cell markers CD44v6 and ABCG2 in tongue cancer: Effect of neoadjuvant chemotherapy on local recurrence
}

\author{
SOUICHI YANAMOTO ${ }^{1}$, SHIN-ICHI YAMADA ${ }^{1}$, HIDENORI TAKAHASHI ${ }^{1}$, TOMOFUMI NARUSE ${ }^{1}$, \\ YUKI MATSUSHITA $^{1}$, HISAZUMI IKEDA ${ }^{2}$, TAKESHI SHIRAISHI ${ }^{2}$, SACHIKO SEKI $^{3}$, \\ SHUICHI FUJITA $^{3}$, TOHRU IKEDA ${ }^{3}$, IZUMI ASAHINA ${ }^{2}$ and MASAHIRO UMEDA ${ }^{1}$ \\ Departments of ${ }^{1}$ Clinical Oral Oncology, ${ }^{2}$ Regenerative Oral Surgery, ${ }^{3}$ Oral Pathology and Bone Metabolism, \\ Graduate School of Biomedical Sciences, Nagasaki University, Nagasaki 852-8588, Japan
}

Received September 26, 2013; Accepted November 11, 2013

DOI: $10.3892 /$ ijo.2014.2289

\begin{abstract}
The efficacy of neoadjuvant chemotherapy (NAC) is controversial, and no report supports NAC with a high evidence level. Recently, we showed that a deep surgical margin was resected very close to the tumor site in many NAC-treated oral squamous cell carcinoma patients, suggesting that NAC may lead to local recurrence and poor outcomes. The purpose of this study was to evaluate the effect of NAC on tumor local recurrence using cancer stem cell marker immunohistochemistry. We retrospectively analyzed 89 patients who underwent radical surgery for tongue cancer, and examined the effect of NAC on tumor local recurrence. Cancer stem cell marker (CD44v6 and ABCG2) expression was detected by immunohistochemistry. In our study, the local recurrence rate was $12.4 \%$. CD44v6 and ABCG2 expression was significantly associated with regional lymph node metastasis, pattern of invasion, depth of invasion, perineural invasion and local recurrence, respectively. Tumor local recurrence was a significant independent predictive factor of the 5-year disease specific survival. CD44v6 or ABCG2 positivity in NAC-treated patients was significantly associated with tumor local recurrence. It was suggested that local recurrence in NAC-treated cases is associated with cancer stem-like cells. We propose that NAC leads to the selection and/or residue of more aggressive cancer stem-like cells.
\end{abstract}

\section{Introduction}

Oral squamous cell carcinoma (OSCC) represents $\sim 1-3 \%$ of all human cancers, and is the 6th most frequent cancer in the world $(1,2)$. OSCC continues to show a poor prognosis and

Correspondence to: Dr Souichi Yanamoto, Department of Clinical Oral Oncology, Graduate School of Biomedical Sciences, Nagasaki University, 1-7-1 Sakamoto, Nagasaki 852-8588, Japan

E-mail: syana@nagasaki-u.ac.jp

Key words: tongue cancer, neoadjuvant chemotherapy, cancer stem cell, CD44v6, ABCG2 remains a lethal disease for $>50 \%$ of cases diagnosed annually (3). In particular, its local recurrence is strongly related to a poor prognosis (4-6). Even when the surgical margins are diagnosed as tumor free by histopathological examination, the local recurrence rate is still $6.9-22.0 \%(4,5,7,8)$. There are two explanations for the mechanism of local recurrence (9). First, there is the possibility that residual cancer cells remain undetected in the surgical margins. In the majority of these cases routine histopathological investigation of the resection specimen will detect tumor tissue at the resection margin, making it likely that residual cancer is the cause of local recurrence. However, the number of residual cancer cells is too small to be detected by routine histopathology, a phenomenon also known as minimal residual cancer (MRC) $(9,10)$. Secondly, it is possible that tumor-related mucosal precursor lesions, 'fields' of genetically altered cells may be left behind, and these might give rise to new invasive carcinomas. A subgroup of these fields can be recognized clinically as leukoplakia or erythroplakia, but the majority can only be diagnosed by conventional histology, immunohistochemical staining, or genetic methods $(9,11,12)$.

Recently, we showed that the deep surgical margin was resected very close to the tumor site in many neoadjuvant chemotherapy (NAC)-treated OSCC patients, suggesting that NAC may lead to local recurrence and poor outcomes $(6,13)$. In these studies $(6,13)$, therefore, we speculated that the most invasive front became unclear after modification by NAC, which may have resulted in resection with an insufficient safety margin. Moreover, we estimated that not only the mechanism for the closed margin but also the residual cancer cells after NAC were involved in the mode of local recurrence. Most recently, some researchers reported that these residual cancer cells after NAC possess the characteristics of cancer stem cells (CSC) $(14,15)$. Yoshikawa et al (15) showed that CD44-expressing cancer cells in the clinical samples of human head and neck cancers selectively survive and increase in number after NAC. CD44 is a single-pass type I transmembrane glycoprotein and functions as a cellular adhesion molecule for hyaluronic acid, a major component of the extracellular matrix $(16,17)$. Expression of CD44, especially 
variant isoforms (CD44v6), is highly expressed in CSCs derived from solid tumors, including brain (18), colon (19), pancreas (20) and head and neck (21) cancer. ATP-binding cassette, subfamily G, member 2 (ABCG2), is a member of the ABC transporter protein superfamily that produces multiple drug-resistant cancer. It is also known as a molecular determinant for maintaining the side population (SP) phenotype in stem cells (22). This phenotype has been observed in OSCC $(23,24)$. Increasing evidence therefore suggests that ABCG2 is recognized as a CSC marker.

However, it is unclear whether NAC is associated with the expression of CD44v6 or ABCG2, or whether the expression of these CSC markers is clinically important in NAC-surviving cells (MRC cells after NAC). We therefore investigated whether NAC is associated with the expression of CD44v6 and ABCG2 using immunohistochemical staining, and evaluated the association with clinicopathological factors and the clinical outcome of oral tongue squamous cell carcinoma (OTSCC) patients.

\section{Materials and methods}

Patients. We retrospectively reviewed the records of 89 patients who underwent radical surgery for OTSCC between January 2001 and December 2011. The study cohort included patients with histologically confirmed diagnoses of OTSCC and a minimum follow-up of 12 months. All study patients underwent extensive pretreatment evaluations, including blood chemistry, complete blood cell count, chest X-ray, computed tomography (CT) and/or magnetic resonance imaging (MRI) of the head and neck area, and thoracoabdominal CT, and provided informed consent to participate in the study. Data collected included demographic information, site, TNM stage at diagnosis, tumor histologic grade, pattern of tumor invasion, treatment, depth of tumor invasion, status of the margin, histopathological response after NAC, recurrence, and the adjuvant therapy used for disease control.

Tumor stage was classified according to the TNM classification of the International Union against Cancer. Tumor histologic grade was defined according to the WHO classification. Pattern of invasion (POI) was examined at the host/ tumor interface. POI types 1-4 have been previously defined by Bryne et al (25): POI type 1, tumor invasion in a broad pushing manner with a smooth outline. POI type 2 , tumor invasion with broad pushing 'fingers' or separate large tumor islands, with a stellate appearance. POI type 3 , invasive islands of tumor $>15$ cells per island. POI type 4 , invasive tumor islands $<15$ cells per island. This includes cord-like and single cell invasion. The evaluation was performed using biopsy specimens.

Treatment and pathologic examination. In our institute, the modality of surgery alone was preferred for the treatment of patients with oral cancer; however, patients who hesitated to consent to surgical intervention or for whom surgery was not available because of a busy schedule were selected for NAC. During the period of NAC, patients were encouraged to undergo surgery after completion of chemotherapy. All patients who received NAC underwent radical surgery. The regimen of NAC consisted of a combination of cisplatin or carboplatin-based multidrugs. In most patients, modifications of chemotherapy dosages were made for toxicity as indicated using standard criteria.

Primary tumors were excised with $\geq 1.5-\mathrm{cm}$ safety margins (both superficial and deep margins). The extent of surgery was not modified according to the tumor response with NAC. All patients underwent glossectomy with curative intent. Elective neck dissection was not performed routinely in our institutions. Local recurrence was defined as lesions arising in the oral cavity relative to the primary tumor beyond 6 weeks within the first 5 years after the first definitive treatment. Recurrence arising at both the primary site and in the neck was also considered to be local recurrence. Recurrence arising only in the neck was defined as regional recurrence. Recurrence arising either at the primary site or in the neck was defined as locoregional recurrence. Patients with recurrences that were considered potentially curable and operable underwent salvage surgery and radiotherapy. Patients with recurrences that were considered incurable were treated with palliative chemotherapy.

For pathological examination of the invasion depth and surgical margin, all permanent section histological slides of primary resection specimens were reviewed retrospectively. Depth of invasion (DOI) was measured as the infiltrative portion of the tumor which extended below the surface of the adjacent mucosa. The keratin layer was not included in the measurements. Where the epithelium was destroyed (since the majority of the tumor showed ulceration), it was measured after reconstructing a virtual surface. Previous studies demonstrated that DOI $\geq 4 \mathrm{~mm}$ had a predictive value for cervical lymph node metastasis in patients with OTSCC (26-30); therefore, in this study, DOI was classified as $\geq 4$ and $<4 \mathrm{~mm}$. Likewise, regarding the surgical margin, a large cohort study demonstrated that a pathological margin distance $\leq 4 \mathrm{~mm}$ was significantly associated with locoregional recurrence (31); therefore, in this study, the status of the surgical margin was classified as $>4$ and $\leq 4 \mathrm{~mm}$ for the superficial and deep margins, respectively. The degree of histopathological response after NAC was classified into four categories, based on the method in previous studies $(32,33)$ : grade I, $>50 \%$ vital residual tumor cells (VRTC); grade II, 10-50\% VRTC; grade III, nearly complete response (NCR) with $<10 \%$ VRTC; and grade IV, complete response. All pathological assessments were performed by expert pathologists who were unaware of the clinical outcomes.

Immunohistochemistry (IHC). Immunohistochemical staining was performed on $4-\mu \mathrm{m}$-thick formalin-fixed, paraffin-embedded tissue sections. Deparaffinized sections in xylene were soaked in $10 \mathrm{mM}$ citrate buffer (pH 6.0) and placed in an autoclave at $121^{\circ} \mathrm{C}$ for $5^{\circ} \mathrm{min}$ for antigen retrieval. Endogenous peroxidase was blocked using $0.3 \%$ $\mathrm{H}_{2} \mathrm{O}_{2}$ in methanol for $30 \mathrm{~min}$. Immunohistochemistry was performed by the EnVision method (EnVision ${ }^{+}$; Dako, Glostrup, Denmark). The primary antibodies used were directed against CD44v6 (VFF-18; Abcam, Cambridge, UK; 1:100 dilution) and ABCG2 (BXP-21; Abcam; 1:50 dilution). The sections were incubated with the antibodies overnight at $4^{\circ} \mathrm{C}$. Reaction products were visualized by immersing the sections in diaminobenzidine (DAB) solution, and the 
Table I. Demographic characteristics of 89 patients with oral tongue squamous cell carcinoma.

\begin{tabular}{lr}
\hline Characteristics & No. of cases ( \\
\hline Gender & \\
Male & $50(56.2)$ \\
Female & $39(43.8)$ \\
Age & \\
$\geq 64$ & $48(53.9)$ \\
$\leq 63$ & $41(46.1)$ \\
pT stage & \\
T1 & \\
T2 & $39(43.8)$ \\
T3 & $43(48.4)$ \\
T4 & $5(5.6)$ \\
pN stage & $2(2.2)$ \\
N0 & \\
N1 & \\
N2 & $63(70.8)$ \\
N3 & $12(13.5)$ \\
\end{tabular}

Histological grade

Well

$82(92.1)$

Moderately

5 (5.6)

Poorly

POI

1

2

$6 \quad(6.7)$

25 (28.2)

40 (44.9)

3

$18(20.2)$

Treatment

Surgery alone

67 (75.3)

$\mathrm{NAC}+$ surgery

22 (24.7)

DOI

$<4 \mathrm{~mm}$

55 (61.8)

$\geq 4 \mathrm{~mm}$

34 (38.2)

Surgical margin

$>4 \mathrm{~mm}$

68 (76.4)

$\leq 4 \mathrm{~mm}$

$21(23.6)$

Perineural invasion

No

65 (73.0)

Yes

24 (27.0)

Local recurrence

No

78 (87.6)

Yes

$11(12.4)$

POI, pattern of invasion; NAC, neoadjuvant chemotherapy; DOI, depth of invasion.
Table II. Neoadjuvant chemotherapy regimen and disease stage of oral tongue squamous cell carcinoma.

\begin{tabular}{lrrccc}
\hline Treatment & Stage I & Stage II & Stage III & Stage IV & Total \\
\hline Surgery alone & 35 & 23 & 4 & 4 & 67 \\
NAC + surgery & 2 & 11 & 5 & 4 & 22 \\
NAC regimen & & & & & \\
CDDP & 1 & 1 & 0 & 0 & 2 \\
CDDP/S-1 & 0 & 7 & 1 & 1 & 9 \\
CBDCA/5-FU & 1 & 3 & 4 & 3 & 11 \\
\hline
\end{tabular}

NAC, neoadjuvant chemotherapy.

samples were counterstained with Myer's hematoxylin and mounted.

The membranous expression of CD44v6 or ABCG2 was estimated by immunopositivity mainly in the tumor invasive front closed surgical margin. The average number of stained cells in 5 visual fields was regarded as the percent ratio of positively stained cells in each section. Staining with these antibodies was considered positive if $>10 \%$ of tumor cells stained, because a $10 \%$ cutoff level has been used in several previous studies $(34,35)$.

Statistical analysis. Statistical analyses were performed using StatMate IV (Atms Co., Tokyo, Japan). The categorical data were assessed by the $\chi^{2}$ test or Fisher's exact test, as appropriate. The disease-specific survival (DSS) rate was calculated using the Kaplan-Meier method. Significance was evaluated using the log-rank test. A multiple regression study was performed using Cox proportional hazard analysis. Univariate logistic and multivariate logistic regression analyses were used to identify independent predictive factors for local recurrence. Predictors that were not associated with DSS or local recurrence in univariate analysis were not included in multivariate analysis. $\mathrm{P}<0.05$ was considered significant.

\section{Results}

Patient characteristics. Patient demographic characteristics are summarized in Table I. The male-to-female ratio was 1.28 with 50 male subjects. The mean age at diagnosis was 63.4 years (range, 28-88 years). Twenty-two (24.7\%) patients received NAC. NAC regimens were CDDP (CDDP $10 \mathrm{mg}$ / $\mathrm{m}^{2}$, days $1-5 ; 2$ courses with 2 -week intervals), CDDP/S-1 (S-1 $40 \mathrm{mg} / \mathrm{m}^{2}$ twice a day orally for 21 days, CDDP $60 \mathrm{mg}$ / $\mathrm{m}^{2}$ on day $8 ; 2$ courses with 2 -week interval periods), or CBDCA/5-FU (CBDCA $300 \mathrm{mg} / \mathrm{m}^{2}$ on day 1, 5-FU $600 \mathrm{mg}$ on days 1-5; 2 courses with 3 -week interval periods). In NAC-treated patients, 9 patients $(40.9 \%)$ had stage III or IV disease, and 13 patients $(59.1 \%)$ had stage I or II (Table II). Toxicity to NAC was acceptable (CTCAE v. 4.0 grade 1/2) and related to nausea, mucositis and hematologic toxicity.

Local recurrence developed in 11 patients (12.4\%) during the follow-up period. The mean follow-up period was 44.7 months for the whole series (range, 3-125 months). 


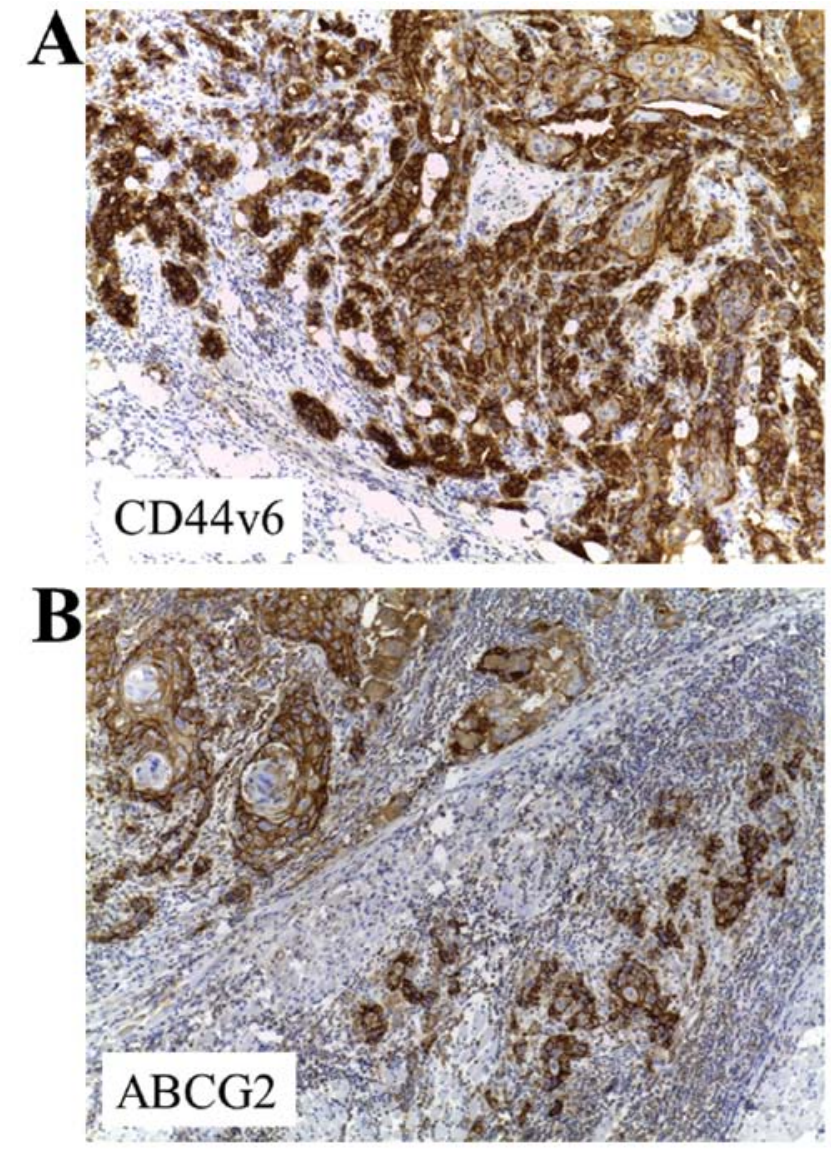

Figure 1. Representative CD44v6 and ABCG2 expression in oral tongue squamous cell carcinoma (x10). (A) CD44v6 was mainly immunostained in the cell membrane or cytoplasm near the cell membrane. (B) ABCG2 was mainly immunostained in the cell membrane.

CD44v6 and ABCG2 expression in OTSCC by IHC. The positive rates of CD44v6 and ABCG2 in 89 OTSCC patients detected by immunohistochemical staining were 24.7 and $13.5 \%$, respectively. Staining for CD44v6 and ABCG2 showed a mixed membranous and cytoplasmic pattern of staining in OTSCC cells (Fig. 1). Both markers were mainly characterized by higher invasive front localization.

Association of CD44v6 and ABCG2 expression with clinicopathological factors and survival. CD44v6 expression was significantly associated with lymph node metastasis, POI, NAC, DOI, perineural invasion, and local recurrence. Meanwhile, ABCG2 was significantly associated with lymph node metastasis, POI, DOI, perineural invasion and local recurrence. Univariate analysis using the log-rank test revealed that T-stage $(\mathrm{P}=0.010)$, lymph node metastasis $(\mathrm{P}=0.003)$, tumor differentiation $(\mathrm{P}=0.011)$, POI $(\mathrm{P}<0.001)$, NAC $(\mathrm{P}<0.001)$, DOI $(\mathrm{P}=0.002)$, perineural invasion $(\mathrm{P}<0.001)$ and local recurrence $(\mathrm{P}<0.001)$ were significantly associated with 5-year DSS (Table III).

Univariate analysis using the log-rank test and KaplanMeier method showed that CD44v6 and ABCG2 expression was significantly associated with 5-year DSS (Fig. 2, $\mathrm{P}<0.001$, respectively). Predictors that were associated with 5-year DSS in univariate analysis were included in the Cox proportional

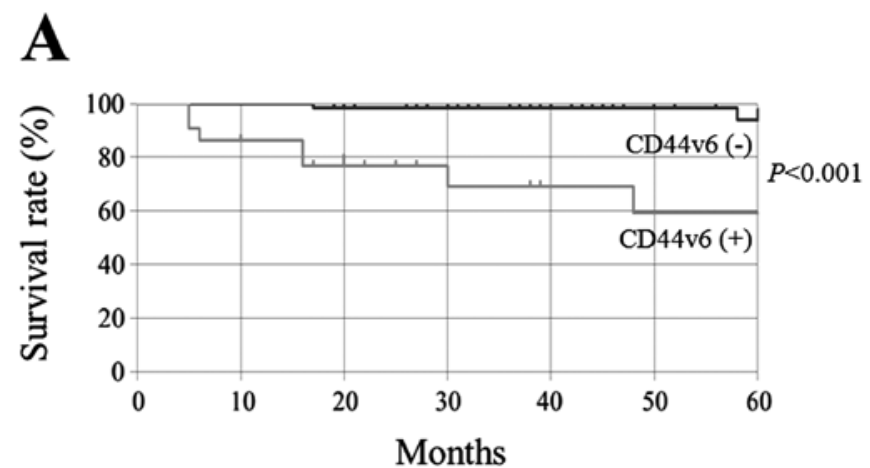

\section{B}

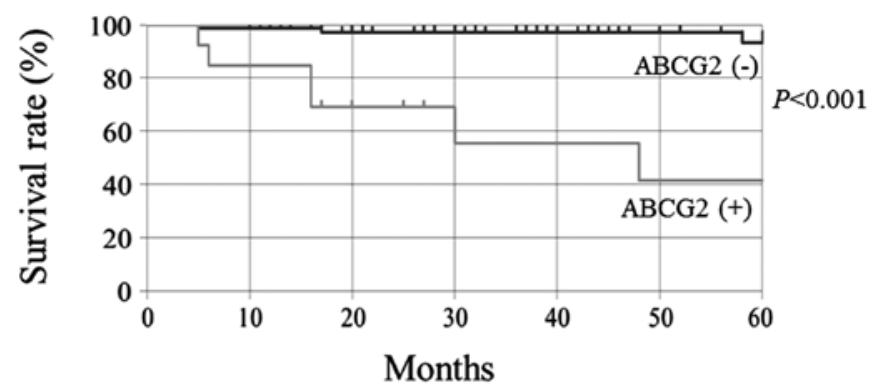

Figure 2. Kaplan-Meier survival curves of the 5-year disease-specific survival (DSS) rate. (A) The 5-year DSS rate of CD44v6-positive patients was significantly shorter than that of $C D 44 \mathrm{v} 6$-negative patients $(\mathrm{P}<0.001)$. (B) Likewise, the 5-year DSS rate of ABCG2-positive patients was significantly shorter than that of ABCG2-negative patients $(\mathrm{P}<0.001)$.

hazard model, and this multivariate analysis showed that local recurrence was only one independent predictor of 5-year DSS in OTSCC patients. The odds ratio of local recurrence was 17.54 (95\% confidence interval, 0.36-5.37; $\mathrm{P}=0.025$ ).

Association with local recurrence. Univariate logistic analysis showed a significant association of tumor local recurrence with POI $(\mathrm{P}<0.001)$, NAC $(\mathrm{P}=0.038)$, DOI $(\mathrm{P}=0.029)$, surgical margin $(\mathrm{P}=0.003), \mathrm{CD} 44 \mathrm{v} 6(\mathrm{P}<0.001)$ and ABCG2 $(\mathrm{P}=0.008)$ (Table IV). Multivariate logistic regression was performed for each predictor of the local recurrence and revealed that only the surgical margin (odds ratio, 10.58; 95\% confidence interval, 1.75-63.89; $\mathrm{P}=0.010$ ) was an independent factor for local recurrence.

Association of NAC with cancer stem cell markers (CD44v6 and $A B C G 2$ ). As mentioned above, the positive rate of CD44v6 in NAC-treated patients was significantly higher than that in surgery alone-treated patients. Likewise, the local recurrence rate in NAC-treated patients was significantly higher than that in surgery alone-treated patients.

Therefore, to examine the effect of NAC on local recurrence, the relationship between cancer stem cell markers (CD44v6 and ABCG2) and local recurrence was analyzed by subgroup assay. There was a significant correlation between local recurrence with $\mathrm{CD} 44 \mathrm{v} 6$ or ABCG2 positivity in the NAC-treated group but not the surgery alone-treated group (Table V). Then, the correlation of the degree of histopathological response after NAC with local recurrence or these 
Table III. Association of CD44v6 and ABCG2 expression with clinicopathological factors and survival.

\begin{tabular}{|c|c|c|c|c|c|c|c|c|c|}
\hline \multirow[b]{2}{*}{ Characteristics } & \multirow[b]{2}{*}{$\mathrm{n}$} & \multicolumn{2}{|c|}{ CD44v6 } & \multirow[b]{2}{*}{ P-value } & \multicolumn{2}{|c|}{ ABCG2 } & \multirow[b]{2}{*}{ P-value } & \multirow[b]{2}{*}{ 5-year DSS (\%) } & \multirow[b]{2}{*}{ P-value } \\
\hline & & + & - & & + & - & & & \\
\hline Gender & & & & 0.417 & & & 0.305 & & 0.422 \\
\hline Male & 50 & 14 & 36 & & 9 & 41 & & 84.3 & \\
\hline Female & 39 & 8 & 31 & & 4 & 35 & & 86.2 & \\
\hline Age & & & & 0.122 & & & 0.231 & & 0.222 \\
\hline$\geq 64$ & 48 & 15 & 33 & & 9 & 39 & & 83.2 & \\
\hline$\leq 63$ & 41 & 7 & 34 & & 4 & 37 & & 88.7 & \\
\hline $\mathrm{pT}$ & & & & 0.505 & & & 0.980 & & 0.010 \\
\hline $\mathrm{T} 1+\mathrm{T} 2$ & 82 & 21 & 61 & & 12 & 70 & & 90.0 & \\
\hline $\mathrm{T} 3+\mathrm{T} 4$ & 7 & 1 & 6 & & 1 & 6 & & 47.6 & \\
\hline $\mathrm{pN}$ & & & & $<0.001$ & & & $<0.001$ & & 0.003 \\
\hline N0 & 63 & 8 & 55 & & 4 & 59 & & 94.5 & \\
\hline $\mathrm{N} 1+\mathrm{N} 2+\mathrm{N} 3$ & 26 & 14 & 12 & & 9 & 17 & & 63.9 & \\
\hline Differentiation & & & & 0.806 & & & 0.276 & & 0.011 \\
\hline Well & 82 & 20 & 62 & & 11 & 71 & & 88.4 & \\
\hline Moderate + poor & 7 & 2 & 5 & & 2 & 5 & & 57.1 & \\
\hline POI & & & & $<0.001$ & & & $<0.001$ & & $<0.001$ \\
\hline $1+2+3$ & 71 & 7 & 64 & & 5 & 66 & & 89.4 & \\
\hline 4 & 18 & 15 & 3 & & 8 & 10 & & 69.1 & \\
\hline Treatment & & & & 0.042 & & & 0.214 & & $<0.001$ \\
\hline Surgery alone & 67 & 13 & 54 & & 8 & 59 & & 96.0 & \\
\hline NAC + surgery & 22 & 9 & 13 & & 5 & 17 & & 63.3 & \\
\hline DOI & & & & $<0.001$ & & & $<0.001$ & & 0.002 \\
\hline$<4 \mathrm{~mm}$ & 55 & 4 & 51 & & 2 & 53 & & 98.0 & \\
\hline$\geq 4 \mathrm{~mm}$ & 34 & 18 & 16 & & 11 & 23 & & 70.2 & \\
\hline Surgical margin & & & & 0.640 & & & 0.510 & & 0.077 \\
\hline$>4 \mathrm{~mm}$ & 68 & 16 & 52 & & 9 & 59 & & 86.2 & \\
\hline$\leq 4 \mathrm{~mm}$ & 21 & 6 & 15 & & 4 & 17 & & 81.0 & \\
\hline Perineural invasion & & & & $<0.001$ & & & 0.018 & & $<0.001$ \\
\hline No & 65 & 10 & 56 & & 6 & 59 & & 96.7 & \\
\hline Yes & 24 & 12 & 12 & & 7 & 17 & & 63.5 & \\
\hline Local recurrence & & & & $<0.001$ & & & 0.002 & & $<0.001$ \\
\hline No & 78 & 14 & 64 & & 8 & 70 & & 88.2 & \\
\hline Yes & 11 & 8 & 3 & & 5 & 6 & & 47.7 & \\
\hline
\end{tabular}

POI, pattern of invasion; NAC, neoadjuvant chemotherapy; DOI, depth of invasion; DSS, disease-specific survival.

cancer stem cell marker expressions was not significant (Table VI).

Immunopositivity of CD44v6 or ABCG2 showed significantly high intensity in NAC-treated patients compared with surgery alone-treated patients. In particular, the immunopositivity of these cancer stem cell markers was apparent at the tumor/host interface in NAC-treated patients with local recur- rence. In contrast, immunopositivity in NAC-treated patients without local recurrence revealed pale or faint staining (Fig. 3).

\section{Discussion}

Locoregional recurrence in patients with OSCC remains a problem and can affect their survival (4-6). In particular, local 
Table IV. Clinicopathological factors and CD44v6 and ABCG2 expression in relation to local recurrence.

\begin{tabular}{|c|c|c|c|c|c|c|}
\hline \multirow[b]{2}{*}{ Characteristics } & \multirow[b]{2}{*}{$\mathrm{n}$} & \multicolumn{2}{|c|}{ Local recurrence } & \multirow[b]{2}{*}{ Odds ratio } & \multirow[b]{2}{*}{$95 \% \mathrm{CI}$} & \multirow[b]{2}{*}{ P-value } \\
\hline & & $+(\mathrm{n}=11)(\%)$ & $-(n=78)$ & & & \\
\hline Gender & & & & & $0.17-2.17$ & 0.659 \\
\hline Male & 50 & $5(10.0)$ & 45 & 0.61 & & \\
\hline Female & 39 & $6(15.4)$ & 33 & 1.00 & & \\
\hline Age & & & & & $0.29-3.65$ & 0.780 \\
\hline$\geq 64$ & 48 & $6(12.5)$ & 42 & 1.03 & & \\
\hline$\leq 63$ & 41 & $5(12.2)$ & 36 & 1.00 & & \\
\hline pT & & & & & $0.13-11.02$ & 0.662 \\
\hline $\mathrm{T} 1+\mathrm{T} 2$ & 82 & $10(12.2)$ & 72 & 1.00 & & \\
\hline $\mathrm{T} 3+\mathrm{T} 4$ & 7 & $1(14.3)$ & 6 & 1.20 & & \\
\hline $\mathrm{pN}$ & & & & & $0.22-6.89$ & 0.833 \\
\hline No & 63 & $7(11.1)$ & 56 & 1.00 & & \\
\hline $\mathrm{N} 1+\mathrm{N} 2+\mathrm{N} 3$ & 26 & $4(15.4)$ & 22 & 1.24 & & \\
\hline Differentiation & & & & & $0.55-19.24$ & 0.448 \\
\hline Well & 82 & $9(11.0)$ & 73 & 1.00 & & \\
\hline Moderate + poor & 7 & $2(28.6)$ & 5 & 3.24 & & \\
\hline POI & & & & & $2.67-42.5$ & $<0.001$ \\
\hline $1+2+3$ & 71 & $4 \quad(5.6)$ & 67 & 1.00 & & \\
\hline 4 & 18 & $7(38.9)$ & 11 & 10.66 & & \\
\hline Treatment & & & & & $1.26-17.20$ & 0.038 \\
\hline Surgery alone & 67 & $5(9.1)$ & 62 & 1.00 & & \\
\hline NAC + surgery & 22 & $6(21.7)$ & 16 & 4.65 & & \\
\hline DOI & & & & & $1.30-21.80$ & 0.029 \\
\hline$<4 \mathrm{~mm}$ & 55 & $3(5.5)$ & 52 & 1.00 & & \\
\hline$\geq 4 \mathrm{~mm}$ & 34 & $8(21.1)$ & 26 & 5.33 & & \\
\hline Surgical margin & & & & & $2.06-31.10$ & 0.003 \\
\hline$>4 \mathrm{~mm}$ & 68 & $4 \quad(5.9)$ & 64 & 1.00 & & \\
\hline$\leq 4 \mathrm{~mm}$ & 21 & $7(33.3)$ & 14 & 8.00 & & \\
\hline Perineural invasion & & & & & $0.71-9.44$ & 0.267 \\
\hline No & 65 & $6 \quad(9.2)$ & 59 & 1.00 & & \\
\hline Yes & 24 & $5(20.8)$ & 19 & 2.59 & & \\
\hline CD44v6 & & & & & $2.87-51.84$ & $<0.001$ \\
\hline Positive & 22 & $8(36.4)$ & 14 & 12.19 & & \\
\hline Negative & 67 & $3(4.5)$ & 64 & 1.00 & & \\
\hline ABCG2 & & & & & $1.81-29.40$ & 0.008 \\
\hline Positive & 13 & $5(38.5)$ & 8 & 7.29 & & \\
\hline Negative & 76 & $6 \quad(7.9)$ & 70 & 1.00 & & \\
\hline
\end{tabular}

CI, confidence interval; POI, pattern of invasion; NAC, neoadjuvant chemotherapy; DOI, depth of invasion.

recurrence is a high potential risk factor for survival $(4,6)$. Correspondingly, in our study, tumor local recurrence was only one predictive factor of the 5-year DSS rate by multivar- iate analysis. Previous studies reported that local recurrence occurred in $6.9-22.0 \%(4,5,7,8)$, similar to the $12.4 \%$ rate in the present series. Several studies have demonstrated that demo- 
Table V. Relationship between CD44v6, ABCG2 and local recurrence by subgroup (surgery alone and NAC) assay.

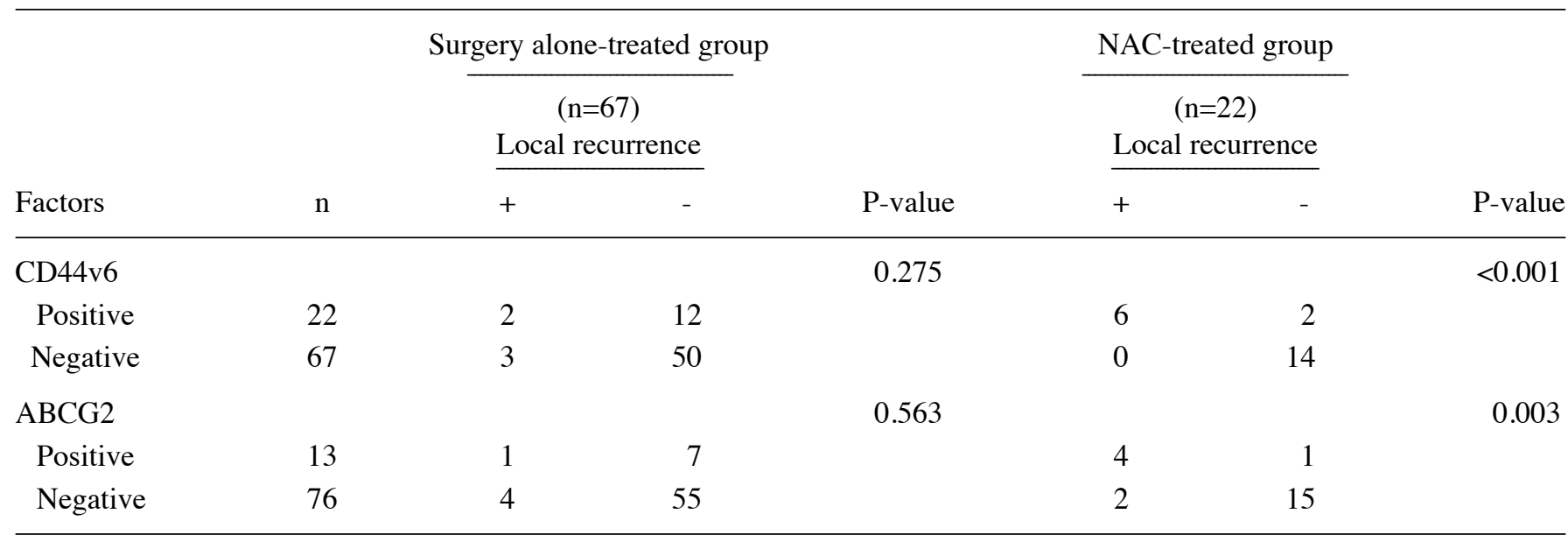

NAC, neoadjuvant chemotherapy.
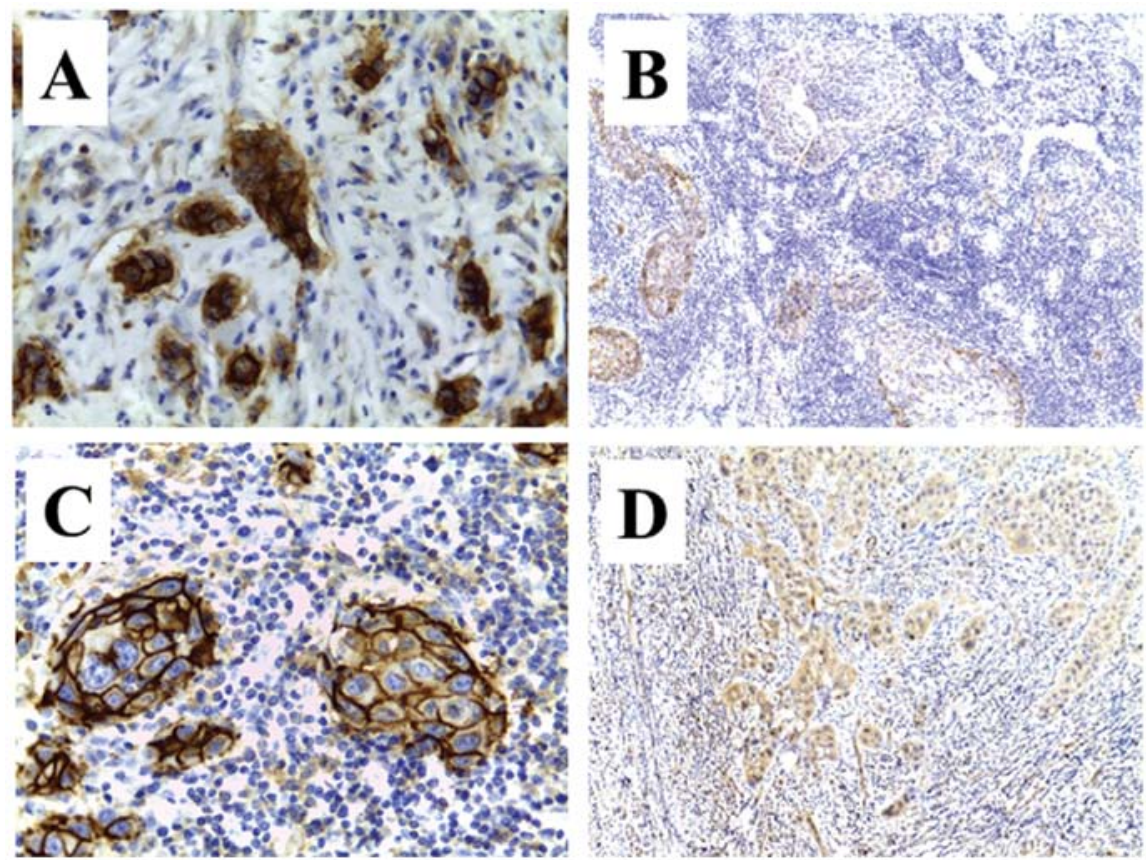

Figure 3. Immunopositivity of CD44v6 or ABCG2 showed significantly high intensity in NAC-treated patients compared with surgery alone-treated patients. (A) CD44v6 immunopositivity was located in tumor satellites at the tumor/host interface in NAC-treated patients with local recurrence (x40), (B) whereas CD44v6 expression in NAC-treated patients without local recurrence was focal or faint. A representative case of CD44v6-negative expression (x10) (C). (D) Likewise, ABCG2 immunopositivity in NAC-treated patients with local recurrence revealed high intensity compared with that in NAC-treated patients without local recurrence.

graphic variables such as site, TNM stage, and simultaneous disorder, and pathological variables such as DOI, surgical margin, and POI could be reliable parameters for local recurrence (4-7). In our study, POI, NAC, DOI, surgical margin, and perineural invasion were significantly associated with local recurrence, and in particular, the surgical margin $(\leq 4 \mathrm{~mm})$ was a predictive factor of local recurrence. Based on data similar to these results, we previously proposed that the most invasive front became unclear after modification by NAC, which may have resulted in resection with an insufficient safety margin $(6,13)$. It was also hypothesized in the mechanism of tumor local recurrence that this elevated risk may be due to unde- tected tumor cells present in surgical margins thought to be negative by conventional light microscopy (9-11). Recently, to improve the detection of residual cancer cells or tumor-related precursor lesions remaining in histologically negative margins, molecular margin analysis was proposed and has now been used to observe and predict local recurrence of head and neck cancers $(9,12,36)$. We therefore focused on the expression of molecules relevant to CSCs in tumor satellites at the tumor/ host interface.

The presence of CSCs in the primary tumor appears to have prognostic significance in various types of cancer including OSCC $(24,37,38)$. Since the concept of CSC has been 
Table VI. Correlation of the histopathological response after NAC with local recurrence or cancer stem cell marker expressions.

Histopathological response

\begin{tabular}{lcccccc}
\hline & \multicolumn{6}{c}{ Histopathological response } \\
\cline { 5 - 6 } Factors & $\mathrm{n}$ & $\mathrm{I}$ & $\mathrm{II}$ & $\mathrm{III}$ & $\mathrm{IV}$ & P-value \\
\hline Local recurrence & & & & & & 0.422 \\
+ & 6 & 1 & 3 & 2 & 0 & \\
- & 16 & 1 & 14 & 1 & 0 & \\
CD44v6 & & & & & & 0.133 \\
Positive & 8 & 2 & 4 & 2 & 0 & \\
Negative & 14 & 0 & 13 & 1 & 0 & \\
ABCG2 & & & & & & 0.816 \\
Positive & 5 & 1 & 3 & 1 & 0 & \\
Negative & 17 & 1 & 14 & 2 & 0 & \\
\hline
\end{tabular}

NAC, neoadjuvant chemotherapy.

proposed to explain tumor cell heterogeneity, some research has suggested that current therapies fail to prevent cancer relapse and metastasis because of a small, surviving population of CSC (37-41). We previously demonstrated that the side population cells isolated from an oral cell line possessed the characteristics of cancer stem cells, chemoresistance, and high proliferation ability (24). Moreover, in another study, we estimated that not only the mechanism for the closed margin but also residual cancer cells exhibiting the characteristic of CSC after NAC are involved in the mode of local recurrence (13). We focused on the expressions of CD44v6 and ABCG2 as CSC markers in OTSCC, because we hypothesized that residual cancer cells exhibiting the characteristics of CSC would be relevant to the mechanism of local recurrence in NAC-treated OTSCC patients.

CD44 is a cell surface glycoprotein known to be a hyaluronate receptor, which may function in lymphocyte homing and cancer metastasis $(16,17)$. CD44 is encoded by a single gene which contains 20 exons and is located on the short arm of chromosome 11 (11p13) (16). Exons 1-5 and 16-19 are spliced together to form the transcript for the standard isoform of CD44 (CD44s), which is expressed in a wide range of normal tissues as well as in tumors of ectodermal origin (16). Alternative splicing of exons 6-15 (variant exons 1-10) gives rise to numerous variant forms of CD44 (CD44v), in which an additional segment encoded by one or more of the variant exons is inserted in the extracellular domain of CD44s, which is encoded by exons $1-5$ and exons $16-20(16,42,43)$. The variant 6 isoform (CD44v6), in particular, is associated with several cancer types but not in somatic cells (15,18-21). Moreover, CD44v6 is widely known as a marker of CSCs $(18,21,36,44-$ 46). Recently, Zhao et al reported that immunohistochemical assessment of CD44v6 in a negative surgical margin would be a valuable approach for predicting recurrence and survival after curative surgery for laryngeal cancer (36). Some authors showed that CD44v6 could be associated with cancer progres- sion, metastasis, and recurrence $(36,46)$. Our data indicate that CD44v6 expression is associated with regional lymph node metastasis, POI, NAC, DOI, perineural invasion, and local recurrence in OTSCC. CD44v6-positive patients revealed a significant poor survival rate compared with CD44v6-negative patients. Furthermore, CD44v6 expression was a predictive factor for local recurrence (odds ratio, 12.19), as well as diffuse POI, NAC modality, deep ( $\geq 4 \mathrm{~mm})$ DOI, closed $(\leq 4 \mathrm{~mm})$ surgical margin in univariate logistic analysis. These results support the idea that CD44v6-positive cancer cells exhibiting the characteristics of CSC take part in the mechanism of local recurrence, and CD44v6 is a useful marker of tumor progression in OTSCC.

ABCG2 has been shown to be a major mediator of the SP phenotype, which refers to a transmembrane transporter that carries out the important biological function of the efflux of multiple endogenous and exogenous substances out of cells $(22,23)$. Elevated expression of ABCG2 has been observed in a number of putative CSCs from various cancers $(24,34,35)$. AGCG2 is a well-known marker useful for identifying and isolating CSCs $(22-24,34,35,47)$. In our study, ABCG2 expression was $14.6 \%$ in OTSCC patients, similar to the $12.4 \%$ in esophageal squamous cell carcinoma (35). Our data indicate that ABCG2 expression is associated with regional lymph node metastasis, POI, DOI, perineural invasion, and local recurrence in OTSCC. ABCG2-positive patients revealed a significant poor survival rate compared with ABCG2-negative patients. Furthermore, ABCG2 expression was a predictive factor for local recurrence (odds ratio, 7.29) in univariate logistic analysis. As well as CD44v6, these results support the idea that ABCG2 is relevant to the mechanism of local recurrence in OTSCC.

Previously, we reported that the surgical margins were resected very close to the site in many NAC-treated cases, suggesting that NAC would lead to local recurrence and a poor outcome in OSCC $(6,13)$. Correspondingly, in this current study, we demonstrated that NAC was a significant predictive factor for local recurrence. NAC, which is the use of systemic chemotherapy before definitive surgery and/or radiotherapy, is an attractive approach in the management of HNSCC. The benefits of NAC for patients with locally advanced head and neck cancer, as demonstrated by several clinical studies, include a reduction in distant metastasis and the preservation of organ function (48-51); however, NAC has failed to demonstrate any significant improvement of survival in several randomized control trials $(49,50)$. The efficacy of NAC is controversial, and no report supports NAC with a high evidence level.

Interestingly, in this study, the positive rate of CD44v6 in NAC-treated cases was significantly higher than that in surgery alone-treated cases. Moreover, there was significant correlation between local recurrence and CD44v6 positivity in the NAC-treated group. On the other hand, this correlation in the surgery-alone group was not significant. These results indicate the possibility that the upregulation of CD44v6 expression may play an important role in local recurrence in patients with OTSCC who are receiving NAC. Several studies have reported that NAC could significantly modify the expression of CSC markers such as CD44v6, CD133, Nanog, Oct4, or EpCAM in various types of cancers $(15,52-55)$. In rectal cancer, some authors demonstrated that CD133 expression in residual cancer 
cells after chemoradiotherapy was significantly higher than matched pre-chemoradiotherapy CD133 expression in biopsy specimens (53-55). In HNSCC, Yoshikawa et al reported that the relative area occupied by CD44v-expressing cells was significantly larger in the tumors of patients who received NAC than in those of patients who did not, suggesting that CD44-positive HNSCC cells are resistant to chemotherapy compared with CD44v-negative cells (15). Thus, we conclude that CD44v6 could be induced by NAC, and CD44v6expressing residual cancer cells exhibiting the characteristics of CSC could be relevant to the mechanism of local recurrence in NAC-treated OTSCC patients. On the other hand, in this study, there was no significant difference in the positive rate of ABCG2 between NAC-treated cases and surgery alone-treated cases. In subgroup analysis, however, there was a significant correlation between local recurrence and ABCG2 positivity in the NAC-treated group, but not in the surgery alone-treated group. These results suggest that ABCG2-expressing cells are resistant to NAC, thereby increasing the fraction of $\mathrm{ABCG} 2-$ expressing cells so that ABCG2-expressing residual cancer cells would be relevant to the mechanism of local recurrence in NAC-treated OTSCC patients. Some studies have also shown that patients whose disease responded to NAC had better local control and survival than those who received ineffective NAC $(48,51)$. In our study, however, the relationship between the histological response after NAC and local recurrence or CSC marker expression was unclear.

Taken together, the expression of CSC markers (CD44v6 and ABCG2) was correlated with lymph node metastasis, tumor progression, and local recurrence, thereby decreasing survival. We conclude that the expression of these CSC markers plays an important role in local recurrence in patients with OTSCC who are receiving NAC. Therefore, we propose that NAC leads to the selection and/or residue of more aggressive CSCs. Accordingly, at present, we cannot recommend the modality of NAC for patients with OTSCC. Detection of the CD44v6 and ABCG2 status in the surgical margin may contribute to the identification of patients with high risk factors of local recurrence and should be addressed in future studies.

\section{References}

1. Jemal A, Bray F, Center MM, Ferlay J, Ward E and Forman D: Global Cancer Statistics 2011. CA Cancer J Clin 61: 69-90, 2011.

2. Warnakulasuriya S: Global epidemiology of oral and oropharyngeal cancer. Oral Oncol 45: 309-316, 2009.

3. Rogers SN, Brown JS, Woolgar JA, et al: Survival following primary surgery for oral cancer. Oral Oncol 45: 201-211, 2009.

4. Brandwein-Gensler M, Teixeira MS, Lewis CM, et al: Oral squamous cell carcinoma. Histologic risk assessment, but not margin status, is strongly predictive of local disease-free and overall survival. Am J Surg Pathol 29: 167-178, 2005.

5. Kernohan MD, Clark JR, Gao K, Ebrahimi A and Milross CG Predicting the prognosis of oral squamous cell carcinoma after first recurrence. Arch Otolaryngol Head Neck Surg 136: $1235-1239,2010$.

6. Yanamoto S, Yamada S, Takahashi H, et al: Clinicopathological risk factors for local recurrence in oral squamous cell carcinoma. Int J Oral Maxillofac Surg 41: 1195-1200, 2012.

7. Liao CT, Chang JTC, Wang HM, et al: Analysis of risk factors of predictive local tumor control in oral cavity cancer. Ann Surg Oncol 15: 915-922, 2008.

8. Ord RA, Kolokythas A and Reynolds MA: Surgical salvage for local and regional recurrence in oral cancer. J Oral Maxillofac Surg 64: 1409-1414, 2006.
9. Braakhuis BJM, Bloemena E, Leemans CR and Brakenhoff RH: Molecular analysis of surgical margins in head and neck cancer: more than a marginal issue. Oral Oncol 46: 485-491, 2010.

10. Partridge M, Li SR, Pateromichelakis S, et al: Detection of minimal residual cancer to investigate why oral tumors recur despiteseemingly adequate treatment. Clin Cancer Res 6: 2718-2725, 2000.

11. van Houten VMM, Leemans CR, Kummer JA, et al: Molecular diagnosis of surgical margins and local recurrence in head and neck cancer patients: a prospective study. Clin Cancer Res 10: 3614-3620, 2004.

12. Bilde A, von Buchwald C, Dabelsteen E, Therkildsen MH and Dabelsteen S: Molecular markers in the surgical margin of oral carcinomas. J Oral Pathol Med 38: 72-78, 2009.

13. Yanamoto S, Yamada S, Takahashi H, et al: Predictors of locoregional recurrence in T1-2N0 tongue cancer patients. Pathol Oncol Res 19: 795-803, 2013.

14. Kim JJ and Tannock IF: Repopulation of cancer cells during therapy: an important cause of treatment failure. Nat Rev Cancer 5: 516-525, 2005.

15. Yoshikawa $\mathrm{M}$, Tsuchihashi $\mathrm{K}$, Ishimoto $\mathrm{T}$, et al: $\mathrm{xCT}$-expressing tumor cells that are resistant to EGFR-targeted therapy in head and neck squamous cell carcinoma. Cancer Res 73: 1855-1866, 2013.

16. Naor D, Sionov RV and Ish-Shalom D: CD44: structure, function, and association with the malignant process. Adv Cancer Res 71: 241-319, 1997.

17. Nagano O, Okazaki S and Saya H: Redox regulation in stem-like cancer cells by CD44 variant isoforms. Oncogene 32: 5191-5198, 2013.

18. Jijiwa M, Demir H, Gupta S, et al: CD44v6 regulates growth of brain tumor stem cells partially through the AKT-mediated pathway. PLoS One 6: e24217, 2011.

19. Lee HJ, Eom DW, Kang GH, et al: Colorectal micropapillary carcinomas are associated with poor prognosis and enriched in markers of stem cells. Mod Pathol 26: 1123-1131, 2013.

20. Gaviraghi M, Tunici P, Valensin S, et al: Pancreatic cancer spheres are more than just aggregates of stem marker-positive cells. Biosci Rep 31: 45-55, 2011.

21. Mack B and Gires O: CD44s and CD44v6 expression in head and neck epithelia. PLoS One 3: e3360, 2008.

22. Zhou S, Schuetz JD, Bunting KD, et al: The ABC transporter $\mathrm{Bcrp} 1 / \mathrm{ABCG} 2$ is expressed in a wide variety of stem cells and is a molecular determinant of the side-population phenotype. Nat Med 67: 1028-1034, 2001.

23. Zhang P, Zhang Y, Mao L, Zhang Z and Chen W: Side population in oral squamous cell carcinoma possesses tumor stem cell phenotypes. Cancer Lett 277: 227-234, 2009.

24. Yanamoto S, Kawasaki G, Yamada S, et al: Isolation and characterization of cancer stem-like side population cells in human oral cancer cells. Oral Oncol 47: 855-860, 2011

25. Bryne M, Koppang HS, Lilleng R and Kjaerheim A: Malignancy grading of the deep invasive margins of oral squamous cell carcinoma has high prognostic value. J Pathol 166: 375-381, 1992.

26. Asakage T, Yokose T, Mukai K, et al: Tumor thickness predicts cervical metastasis in patients with stage I/II carcinoma of the tongue. Cancer 82: 1443-1448, 1998.

27. Kurokawa H, Yamashita Y, Takeda S, Zhang M, Fukuyama $\mathrm{H}$ and Takahashi T: Risk factors for late cervical lymph node metastases in patients with stage I or II carcinoma of the tongue. Head Neck 24: 731-736, 2002.

28. Lim SC, Zhang S, Ishii G, et al: Predictive markers for late cervical metastasis in stage I and II invasive squamous cell carcinoma of the oral tongue. Clin Cancer Res 10: 166-172, 2004.

29. Sparano A, Weinstein G, Chalian A, Yodul M and Weber R: Multivariate predictors of occult neck metastasis in early oral tongue cancer. Otolaryngol Head Neck Surg 131: 472-476, 2004.

30. Huang SH, Hwang D, Lockwood G, Goldstein DP and O'Sullivan B: Predictive value of tumor thickness for cervical lymph-node involvement in squamous cell carcinoma of the oral cavity. A meta-analysis of reported studies. Cancer 115: 1489-1497, 2009.

31. Fan KH, Wang HM, Kang CJ, et al: Treatment results of postoperative radiotherapy on squamous cell carcinoma of the oral cavity: coexistence of multiple minor risk factors results in higher recurrence rates. Int J Radiat Oncol Biol Phys 77: 1024-1029, 2010.

32. Schneider PM, Baldus SE, Metzger R, et al: Histomorphologic tumor regression and lymph node metastases determine prognosis following neoadjuvant radiochemotherapy for esophageal cancer. Ann Surg 242: 684-692, 2005. 
33. Bollschweiler E, Besch S, Drebber U, et al: Influence of neoadjuvant chemoradiation on the number and size of analyzed lymph nodes in esophageal cancer. Ann Surg Oncol 17: 31873194, 2010.

34. Ota S, Ishii G, Goto K, et al: Immunohistochemical expression of BCRP and ERCC1 in biopsy specimen predicts survival in advanced non-small-cell lung cancer treated with cisplatin-based chemotherapy. Lung Cancer 64: 98-104, 2009.

35. Hang D, Dong HC, Ning T, Dong B, Hou DL and Xu WG: Prognostic value of the stem cell markers CD133 and ABCG2 expression in esophageal squamous cell carcinoma. Dis Esophagus 25: 638-644, 2012.

36. Zhao H, Ren J, Zhuo X, Ye H, Zou J and Liu S: Prognostic significance of surviving and CD44v6 in laryngeal cancer surgical margins. J Cancer Res Clin Oncol 134: 1051-1058, 2008.

37. Dalerba P, Cho RW and Clarke MF: Cancer stem cells: models and concepts. Annu Rev Med 58: 267-284, 2007.

38. Visvader JE and Lindeman GJ: Cancer stem cells in solid tumours: accumulating evidence and unresolved questions. Nat Rev Cancer 8: 755-768, 2008.

39. Graziano A, d'Aquino R, Tirino V, Desiderio V, Rossi A and Pirozzi G: The stem cell hypothesis in head and neck cancer. J Cell Biochem 103: 408-412, 2008.

40. Prince ME and Ailles LE: Cancer stem cells in head and neck squamous cell cancer. J Clin Oncol 26: 2871-2875, 2008.

41. Sinha N, Mukhopadhyay S, Das DN, Panda PK and Bhutia SK: Relevance of cancer initiating/stem cells in carcinogenesis and therapy resistance in oral cancer. Oral Oncol 49: 854-862, 2013.

42. Screaton GR, Bell MV, Jackson DG, Cornelis FB, Gerth U and Bell JI: Genomic structure of DNA encoding the lymphocyte homing receptor CD44 reveals at least 12 alternatively spliced exons. Proc Natl Acad Sci USA 89: 12160-12164, 1992.

43. Goodison S, Urquidi V and Tarin D: CD44 cell adhesion molecules. Mol Pathol 52: 189-196, 1999.

44. Wang SJ, Wong G, de Heer AM, Xia W and Bourguignon LY: CD44 variant isoforms in head and neck squamous cell carcinoma progression. Laryngoscope 119: 1518-1530, 2009.
45. Yang YM and Chang JW: Bladder cancer initiating cells (BCICs) are among (initiating) cells. Cancer Invest 26: 725-733, 2008.

46. Shi J, Zhou Z, Di W and Li N: Correlation of CD44v6 expression with ovarian cancer progression and recurrence. BMC Cancer 13: 182, 2013.

47. Shen B, Dong P, Li D and Gao S: Expression and function of ABCG2 in head and neck squamous cell carcinoma and cell lines. Exp Ther Med 2: 1151-1157, 2011.

48. Lore JM, Diaz-Ordaz E, Spaulding M, et al: Improved survival with preoperative chemotherapy followed by tumor response for advanced squamous cell carcinoma of the head and neck. Am J Surg 170: 506-511, 1995 .

49. Pignon JP, Bourhis J, Domenge C and Designe L: Chemotherapy added to locoregional treatment for head and neck squamous cell carcinoma: three meta-analyses of updated individual data. Lancet 355: 949-955, 2000.

50. Licitra L, Grandi C, Guzzo M, et al: Primary chemotherapy in resectable oral cavity squamous cell carcinoma: a randomized controlled trial. J Clin Oncol 21: 327-333, 2003.

51. Olasz L, Szalma J, Orsi E, Tornoczky T, Marko T and Nyarady Z: Neoadjuvant chemotherapy: does it have benefits for surgeon in the treatment of advanced squamous cell cancer of the oral cavity? Pathol Oncol Res 16: 207-212, 2010.

52. Costa S, Terzano P, Bovicelli A, et al: CD44 isoform 6 (CD44v6) is a prognostic indicator of the response to neoadjuvant chemotherapy in cervical carcinoma. Gynecol Oncol 80: 67-73, 2001.

53. Saigusa S, Tanaka K, Toiyama Y, et al: Clinical significance of CD133 and hypoxia inducible factor- $1 \alpha$ gene expression in rectal cancer after preoperative chemotherapy. Clin Oncol 23: 323-332, 2011.

54. Kawamoto A, Tanaka K, Saigusa S, et al: Clinical significance of radiation-induced CD133 expression in residual rectal cancer cells after chemoradiotherapy. Exp Ther Med 3: 403-409, 2012.

55. Sprenger T, Conradi L, Beissbarth T, et al: Enrichment of CD133expressing cells in rectal cancers treated with preoperative radiochemotherapy is an independent marker for metastasis and survival. Cancer 119: 26-35, 2013. 